\title{
MAPA CONCEITUAL COMO FERRAMENTA DE APRENDIZAGEM NO ENSINO SUPERIOR
}

\author{
Cristiane Tolentino Machado ${ }^{1}$ \\ Ana Amélia Carvalho
}

\begin{abstract}
RESUMO
Os mapas conceituais têm sido aceitos como instrumentos efetivos para demonstrar os conteúdos aprendidos. Neste estudo os mapas conceituais foram utilizados para verificar a aprendizagem dos estudantes universitários acerca de um artigo científico, destacando-se que tais mapas foram criados com o apoio do software Cmap Tools ou Cmap Cloud. Além disso, foram avaliadas as percepções dos estudantes sobre abordagem instituída, bem como seus aspectos positivos e negativos por meio de questionários. As respostas dos estudantes foram analisadas por percentagem e de acordo com a técnica de análise de conteúdo. Os mapas conceituais foram em geral bem aceitos pelos alunos como uma ferramenta útil para promover a sua aprendizagem e auxiliar na identificação das dificuldades. A maioria cumpriu com sucesso os quesitos avaliados nos mapas conceituais, apesar de mostrarem alguma dificuldade na utilização do software. Perante as potencialidades dos mapas conceituais aliados às tecnologias digitais, deve-se incentivar a adoção de práticas que promovam a aprendizagem significativa e o desenvolvimento de habilidades inerentes ao contexto acadêmico.
\end{abstract}

Palavras-chave: Aprendizagem significativa. Ensino Superior. Estudantes universitários. Mapas conceituais.

\section{CONCEPT MAP AS A LEARNING TOOL IN HIGHER EDUCATION}

\begin{abstract}
Concept maps have been accepted as effective tools to demonstrate the learned contents. In this study, concept maps were used to verify university students' learning about a scientific article being created with the support of Cmap Tools or Cmap Cloud software. In addition, students' perceptions of the implemented approach were evaluated, as well as their positive and negative aspects through questionnaires. Student responses were analyzed by percentage and according to the content analysis technique. Concept maps were generally well accepted by students as a useful tool to promote their learning and to help identify difficulties. Most students successfully met the requirements evaluated in the concept maps, despite indicating some difficulty in using the software. Given the potential of concept maps combined with digital technologies, the implementation of practices that promote meaningful learning and the development of skills inherent to the academic context should be encouraged.
\end{abstract}

Keywords: Concept maps. Higher education. Meaningful learning. Undergraduates.

RECEBIDO EM: 26/5/2019

ACEITO EM: 30/7/2019

\footnotetext{
1 Professora associada na Universidade Federal dos Vales do Jequitinhonha e Mucuri, Diamantina, MG. Licenciada em Ciências Biológicas pela Pontifícia Universidade Católica de Minas Gerais (1995). Mestrado em Morfologia (1999) e Doutorado em Biologia Celular (2004) pela Universidade Federal de Minas Gerais. Atua principalmente nos seguintes temas: tecnologias digitais no ensino superior e divulgação e alfabetização científica no ensino fundamental e médio. http://lattes.cnpq.br/5783316236532224. https://orcid.org/0000-0002-63783300. cristiane.tolentino@gmail.com

${ }^{2}$ Professora catedrática na Faculdade de Psicologia e de Ciências da Educação da Universidade de Coimbra, Portugal. Licenciada em Ensino de Português e de Francês pela Universidade do Minho (1985). Mestrado em Educação pela Universidade de Manchester (1991). Doutorado em Educação na especialidade de Tecnologia Educativa pela Universidade do Minho (1998). Coordena o Laboratório de Tecnologia Educativa (LabTE) da Universidade de Coimbra. http://lattes.cnpq.br/9227547586044410. https://orcid.org/0000-0002-06219447. anaameliac@fpce.uc.pt
} 
A complexidade dos processos de aprendizagem tem demandado aos professores um enfoque mais construtivo, favorecendo a apropriação e produção dos conhecimentos. Há necessidade de se desenvolver habilidades que ultrapassem o domínio cognitivo dos conteúdos e envolvam aspectos como a leitura, compreensão e interpretação de textos científico-tecnológicos por parte dos estudantes universitários, a fim de que tais habilidades os auxiliem na realização de atividades de iniciação científica e no desempenho futuro de sua atuação profissional (SANTOS; QUEIROZ, 2007).

Ao se utilizar os mapas conceituais nas práticas acadêmicas, surge uma possibilidade de se desenvolver nos estudantes a autonomia, corresponsabilidade e permitir uma atuação crítica e comprometida com um aprender significativo. Os mapas conceituais podem ser considerados importantes ferramentas para o acompanhamento da aprendizagem, uma vez que possibilitam uma prática docente diferenciada, tornando o ensino atraente e significativo (CARABETTA JÚNIOR, 2013; RUIZ-MORENO et al., 2007).

Em contextos educativos, os mapas conceituais têm sido usados para a promoção da aprendizagem significativa com efeitos positivos na educação superior. Assim, esta ferramenta contribui para elevar a qualidade do ensino universitário (HAY; KINCHIN; LYGO-BAKER, 2008; NOVAK, 2010; RITCHHART; TURNER; HADAR, 2009).

Estudos demonstraram que os mapas conceituais possibilitaram aos estudantes construírem o seu conhecimento e alcançarem a aprendizagem significativa. Ao construírem os mapas, os estudantes desenvolvem a capacidade crítica e a síntese de ideias (ALMEIDA; MOREIRA, 2008; BES PIÁ; BLASCO-TAMARITI; MUÑOZ-PORTERO, 2011; BITTENCOURT et al., 2013; CARR-LOPEZ et al., 2014; COGO et al., 2009; DUARTE; LOUREIRO; ZUKOWSKY-TAVARES, 2017; ERDEM; YILMAZ; OSKAY, 2009; GONZÁLEZ et al., 2008; KATILIUTE; DAUNORIENE், 2011; OLIVEIRA; SILVA, 2006).

Os mapas elaborados por estudantes podem ser muito úteis, permitindo ao professor acompanhar e avaliar a aprendizagem, tornando-se importantes sinalizadores dos conceitos aprendidos sobre um determinado tema abordado em uma aula ou em textos. São, portanto, uma ótima ferramenta avaliativa, uma vez que se apresentam como instrumentos que revelam os avanços, superações, regulações e autorregulações. A aprendizagem dos conteúdos pode ser evidenciada nos mapas pela organização dos conceitos e qualidade das suas relações. Tornam-se uma ferramenta de meta-aprendizagem na qual o estudante aprende a aprender e a visualizar lacunas na compreensão de determinado conteúdo, o que, por sua vez, favorece a busca de mais informações para esclarecer aquele conceito (ALMEIDA; MOREIRA, 2008; BITTENCOURT et al., 2013; CARABETTA JÚNIOR, 2013; COGO et al., 2009; GREENBERG; WILNER, 2015; RUIZ-MORENO et al., 2007; SOUZA; BORUCHOVITCH, 2010).

Tirando partido das potencialidades dos mapas conceituais, o presente estudo tem como objetivo verificar a aprendizagem acerca de um artigo científico utilizando-se os mapas conceituais criados pelos estudantes universitários em sala de aula, bem como obter as suas percepções sobre a abordagem realizada. Nessa perspectiva, foram analisados os mapas conceituais construídos pelos estudantes baseados em um artigo científico tendo como referência o estudo de Bhusnurmath et al. (2017). Além disso, foram obtidas as suas percepções relativas à abordagem efetivada por meio de questionários com respostas fechadas e abertas. 


\section{MAPAS CONCEITUAIS COM APOIO À TECNOLOGIA}

Mapas conceituais podem também ser feitos em papel e lápis, entretanto, quando associados à tecnologia, oferecem oportunidades de incrementar o seu conteúdo com animações, áudios, vídeos, entre outros. Tem-se constatado um maior envolvimento dos estudantes ao criarem os mapas conceituais utilizando os recursos tecnológicos, além de construírem mapas conceituais mais ricos e criativos (ASAN, 2007; ERDEM; YILMAZ; OSKAY, 2009; LEAUBY; SZABAT; MAAS, 2010; SONNLEITNER et al., 2012; TSAI, LIN; YUAN, 2008; WU; CHEN; HOU, 2016; YEN; LEE; CHEN, 2011). Em um computador, tablet ou celular os mapas conceituais são facilmente construídos. Podem também ser concebidos utilizando-se um software como o Cmap Tools ou o Cmap Cloud, que estão disponíveis on-line. O software facilita a execução dos mapas, permitindo ainda a sua construção de forma colaborativa síncrona ou assíncrona. Tirando partido dos dispositivos móveis dos estudantes, os mapas conceituais tornam-se estratégias adequadas para o ensino em contexto de mobile learning (NOVAK, 2003; YEN; LEE; CHEN, 2011). Com o Cmap Tools ou o Cmap Cloud, os mapas conceituais são criados facilmente. Escrevem-se os conceitos e com os comandos de arrastar e soltar obtêm-se as ligações. A ferramenta ainda permite alterar o aspecto visual do mapa, como cores, formatos, adicionar background. O usuário pode criar mapas conceituais mais elaborados, incorporando aos conceitos os recursos da Internet como imagens, textos, vídeos, páginas da Web ou outros mapas conceituais. Permite ainda exportar os arquivos como imagens, páginas da Web, PDF, XML, armazenar os arquivos e imprimir os mapas conceituais. É possível também, no programa, construir e partilhar os mapas conceituais. São muitas as potencialidades desta ferramenta que permite a construção do conhecimento, a partilha e a colaboração. Dessa forma, ao construir os mapas conceituais é possível integrar várias experiências de aprendizagem (CAÑAS et al., 2004). Mapas conceituais elaborados com recursos tecnológicos apresentam vantagens, como:

São mais fáceis de criar e modificar (ERDOGAN, 2009);

- Proporcionam rápido feedback (NESBIT; ADESOPE, 2006);

- Permitem avaliar a aprendizagem de uma forma mais eficiente (ALMEIDA; MOREIRA, 2008; BITTENCOURT et al., 2013; CARABETTA JÚNIOR, 2013; COGO et al., 2009; GREENBERG; WILNER, 2015; RUIZ-MORENO et al., 2007);

- Possibilitam predefinir alguns conceitos ou ligações para a elaboração dos mapas conceituais pelos estudantes (HO; KUMAR; VELAN, 2014);

- Podem ser elaborados e compartilhados on-line (YEN; LEE; CHEN, 2011; COGO et al., 2009);

- Podem ser incorporados para integrar o ensino, aprendizagem e avaliação em ambientes on-line (HAY; KINCHIN; LYGO-BAKER, 2008; WANG et al., 2017; WU; CHEN; HOU, 2016);

- Tornam as atividades mais motivadoras para os estudantes (ERDEM; YILMAZ; OSKAY, 2009; LEAUBY; SZABAT; MAAS, 2010);

- Podem ser construídos de forma colaborativa em ambiente on-line (WANG et al., 2017). 
Assim sendo, as abordagens relativas à construção de mapas conceituais aliadas às tecnologias digitais em cenários educativos têm sido diversificadas e orientadas para a promoção da aprendizagem ativa e significativa (MIRANDA; MORAIS, 2009).

\section{MAPAS CONCEITUAIS NO ENSINO SUPERIOR}

No Ensino Superior os mapas conceituais são utilizados para diversas finalidades, como auxiliar na sistematização de trabalhos acadêmicos, promoção da aprendizagem significativa, incentivar o trabalho colaborativo, desenvolvimento do pensamento crítico e na resolução de problemas.

a) Auxiliar na análise de dissertação de Mestrado:

Araújo e Formenton (2015) apresentam uma nova proposta de utilização dos mapas conceituais como uma ferramenta de análise na sistematização de informações sobre trabalhos acadêmicos nas etapas de levantamento bibliográfico.

b) Promover a aprendizagem significativa

Ao analisar os mapas conceituais elaborados pelos discentes sobre um tema abordado em uma aula ou a partir da literatura, pode-se ter em consideração os seguintes critérios: quantidade e qualidade de conceitos, níveis de hierarquia, número de inter-relações, palavras de enlace e proposições com significado lógico e estrutura do mapa (BHUSNURMATH et al., 2017). Nos trabalhos analisados os discentes manifestaram-se positivamente sobre a aplicabilidade dos mapas conceituais como ferramentas de aprendizagem, destacando que permitiram evidenciar os conceitos aprendidos pelos discentes, integrar o conhecimento novo, estabelecer inter-relações e prover uma visão integrada do assunto abordado. Possibilitaram também evidenciar as suas dificuldades na aprendizagem dos conteúdos, facilitando o esclarecimento de dúvidas e equívocos. É possível também considerá-los uma estratégia de acompanhamento da aprendizagem ao longo de uma disciplina ou de um curso (ALMEIDA; MOREIRA, 2008; BITTENCOURT et al., 2013; CARABETTA JÚNIOR, 2013; COGO et al., 2009; DUARTE; LOUREIRO; ZUKOWSKY-TAVARES, 2017; FARIAS; FARIAS, 2016; GARCÍA et al., 2012; MOTTA, 2017; RUIZ-MORENO et al., 2007). Ao facilitar a aprendizagem significativa, os mapas conceituais podem ser usados a fim de se alcançar um melhor desempenho acadêmico. Em vários cursos os estudantes que trabalharam com mapas conceituais obtiveram melhor performance nos exames ou testes (ALMEIDA; MOREIRA, 2008; BES PIÁ; BLASCO-TAMARITI; MUÑOZ-PORTERO, 2011; BHUSNURMATH et al., 2017; GARCÍA et al., 2012; MARÉE; VAN BRUGGEN; JOCHEMS, 2013; SILVA et al., 2018; VERONESE et al., 2013).

\section{c) Estimular atividades colaborativas}

Os mapas conceituais podem ser trabalhados de forma coletiva ou individual. $\mathrm{Na}$ maioria dos casos os melhores resultados na aprendizagem consistiam em mapas elaborados coletivamente (GARCÍA et al., 2012; KINCHIN; HAY, 2005). Ao construírem em grupos, os estudantes são estimulados a refletir, partilhar opiniões, dúvidas e a corrigirem equívocos, e assim, construir o seu conhecimento de forma colaborativa (COGO et al., 2009). Em ambientes on-line, os mapas construídos de forma colaborativa foram efetivos para a promoção da aprendizagem e interação entre os estudantes (WANG et 
al., 2017). Em grupos, os estudantes aprendem as vantagens do trabalho colaborativo e adquirem experiência do trabalho em equipe (KALIUTE; DAUNORIENE், 2011). As discussões promoveram a autoavaliação e contribuíram para o desenvolvimento do julgamento clínico (GERDEMAN; LUX; JACKO, 2013).

d) Desenvolver o pensamento crítico

Os mapas conceituais auxiliaram os estudantes a desenvolverem habilidades como a de pensar criticamente, tornando-os mais aptos para a reflexão, tomadas de decisões e para as práticas clínicas (CHEN et al., 2011; RAHNAMA; MARDANI-HAMOOLEH, 2017; SENITA, 2008; VACEK, 2009; WILGS; MCCONNELL, 2008). Os mapas conceituais têm o potencial de se tornarem uma ferramenta útil na avaliação do pensamento crítico no contexto da aprendizagem baseada em problemas (KASSAB et al., 2016; MOK; WHITEHILL; DODD, 2014).

e) Resolver problemas

Ao trabalharem com mapas conceituais os estudantes obtiveram um melhor desempenho na resolução de problemas quando comparados com estudantes que tiveram aulas tradicionais (GONZÁLEZ et al., 2008). Mapas conceituais foram utilizados em uma abordagem de aprendizagem baseada em problemas e mostraram-se efetivos, ocasionando melhores resultados quando comparados ao método tradicional de aprendizagem baseada em problemas (ADDAE; WILSON; CARRINGTON, 2012; CHAN, 2017). No estudo apresentado por Mok, Whitehill e Dodd (2014), os mapas conceituais foram utilizados para o monitoramento do pensamento crítico de estudantes em um currículo de aprendizagem baseada em problemas ao longo de três anos acadêmicos, resultando em um aumento nas pontuações dos mapas conceituais entre $21 \%$ e $33,6 \%$.

\section{CONSTITUINTES DOS MAPAS CONCEITUAIS}

\section{a) Conceitos}

Os conceitos são representados geralmente em círculos ou caixas nos mapas conceituais (NOVAK; CAÑAS, 2006). Novak e Gowin (1984) enfatizaram a identificação e rotulagem de conceitos relevantes para a construção dos mapas conceituais. No mapa conceitual, a identificação de conceitos relevantes ajuda o estudante a organizar as informações e a estabelecer ligações significativas entre conceitos.

b) Palavras de ligação

Nos mapas conceituais são utilizadas linhas para conectar os conceitos com palavras de ligação (NOVAK; GOWIN, 1984). As palavras de ligação devem definir a relação entre os dois conceitos para que ele seja lido como uma afirmação ou proposição válida (NOVAK, 2010). Além disso, Cañas e Novak (2008) especificam que o uso de verbos tende a facilitar o pensamento dinâmico na explicação das relações entre os conceitos.

c) Proposições

A proposição é a unidade básica de um mapa conceitual. Um mapa conceitual pode ser simples e apresentar uma única proposição. No mapa conceitual hierárquico os conceitos podem ser conectados com linhas sem setas, uma vez que as setas são 
redundantes na leitura das proposições na direção de cima para baixo. Para os mapas conceituais apresentados em rede, as linhas que conectam os conceitos são apresentadas com setas apontando as direções para a leitura das proposições. As linhas podem apresentar duas setas, indicando que as proposições podem ser lidas em ambas as direções. Ao ler as proposições os alunos têm a oportunidade de automonitorar seus mapas conceituais, avaliando as proposições e identificando quaisquer ambiguidades ou equívocos (NOVAK; GOWIN, 1984).

d) Cross-links

Cross-links são as relações entre conceitos em diferentes segmentos ou domínios do mapa conceitual (NOVAK; CAÑAS, 2006). Para mapas conceituais com cross-links, os conceitos são conectados como uma rede. Os cross-links que mostram relações válidas entre dois segmentos distintos do mapa conceitual são indicadores de aprendizagem significativa (NOVAK; GOWIN, 1984). Além disso, Novak e Cañas (2006) sugerem que o processo de geração de cross-links envolve altos níveis de desempenho cognitivo.

\section{e) Estrutura}

Kinchin, Hay e Adams (2000) consideraram os três tipos de estruturas de mapas conceituais como em rede, raio e cadeia, respectivamente. Nos mapas conceituais com uma estrutura em rede, que é integrada e hierárquica, um determinado conceito pode ser acessado com flexibilidade por meio de rotas múltiplas. A estrutura em rede revela uma compreensão mais ampla, com conexões entre domínios de conceitos. Nos mapas conceituais com uma estrutura em raio, embora as ligações adicionais possam ser inseridas no conceito principal, torna-se difícil inserir ligações para outros conceitos. Nos mapas conceituais com uma estrutura em cadeia os conceitos adicionais são inseridos em qualquer extremidade da cadeia. Nesta estrutura o acesso a um conceito no centro da cadeia pode ser difícil (KINCHIN; HAY; ADAMS, 2000). Novak e Cañas (2006) consideram que mapas conceituais com estrutura em cadeia ilustram uma má compreensão do material ou uma reestruturação inadequada do mapa.

\section{f) Aparência geral ou layout}

No último passo do processo de construção, os alunos devem garantir que seus mapas conceituais possam ser lidos e compreendidos por outros. Assim, um mapa conceitual pode ser considerado um meio para comunicar o conhecimento adquirido em uma representação de fácil acesso para o visualizador (NOVAK; GOWIN, 1984).

\section{METODOLOGIA}

No estudo realizado no âmbito da disciplina de Tecnologia Educacional do primeiro ano do curso de Licenciatura em Ciências da Educação da Universidade de Coimbra no ano letivo 2016/2017, colocaram-se as seguintes questões de investigação: Podem os mapas conceituais auxiliar os discentes na compreensão de um artigo científico? Quais são as percepções dos discentes sobre a integração dos mapas conceituais nas práticas acadêmicas?

A técnica de coleta dos dados utilizada foi o inquérito por questionário. 


\section{Os Instrumentos}

Antes do início das atividades foi disponibilizado on-line o questionário de caracterização dos estudantes para conhecer as suas experiências e práticas de estudo.

No final os estudantes responderam a um segundo questionário para verificar as suas percepções sobre a estratégia utilizada, bem como seus pontos positivos e negativos. O instrumento integrou a dimensão da percepção dos estudantes sobre a sua experiência com os mapas conceituais, utilizando-se uma escala de tipo Likert com cinco pontos, em que 1- discordo totalmente e 5 - concordo totalmente. 0 questionário termina com duas questões de resposta aberta sobre o que gostaram e o que não gostaram da abordagem adotada.

\section{Tratamento dos Dados dos Questionários}

Foi realizada a análise de percentagem para as questões fechadas dos questionários. As respostas foram agrupadas por: discordância, nem concorda nem discorda ou concordância. As questões de resposta aberta foram analisadas e categorizadas segundo a técnica de análise de conteúdo (AMADO, 2014; COUTINHO, 2013).

\section{A Construção dos Mapas Conceituais}

Em pequenos grupos, os estudantes elaboraram os mapas conceituais com o apoio do software Cmap Tools ou o Cmap Cloud. Os mapas foram construídos baseados no artigo intitulado "Políticas Educativas TIC em Portugal", que caracteriza os três momentos relativamente às políticas educativas na área das Tecnologias da Informação e Comunicação em Portugal, nomeadamente Projeto Minerva, Programa Nónio Século 21 e e-escola (CARVALHO; PESSOA, 2012). Os mapas conceituais eram compartilhados com a professora através do Learning Management System (LMS) para serem avaliados.

\section{A Análise dos Mapas Conceituais}

Tendo como referência o estudo de Bhusnurmath et al. (2017), os mapas conceituais foram analisados a partir de uma escala de 20 pontos contendo os seguintes critérios: (1) organização, (2) interconectividade, (3) clareza e compreensão, (4) precisão da informação e (5) layout (Tabela 1).

Tabela 1 - Escala dos critérios para a análise dos mapas conceituais traduzido e adaptado de Bhusnurmath et al. (2017)

\begin{tabular}{|c|c|c|}
\hline \multicolumn{2}{|l|}{ CRITÉRIOS } & \multirow{2}{*}{$\frac{\text { PONTUAÇÃO }}{2}$} \\
\hline 1 - ORGANIZAÇÃO & Estrutura & \\
\hline & Organização dos conceitos & 1 \\
\hline & Número adequado de conceitos & 1 \\
\hline \multirow[t]{4}{*}{2 - INTERCONECTIVIDADE } & Múltiplas ligações entre os conceitos & 3 \\
\hline & Ligações claras e corretas & 2 \\
\hline & $\begin{array}{l}\text { Ligações entre os conceitos simples e } \\
\text { complexos }\end{array}$ & 2 \\
\hline & Uso de tópicos relevantes & 1 \\
\hline \multicolumn{3}{|c|}{$\begin{array}{l}\text { Contexto \& Educação } \\
\text { Editora Unijuí •ISSN 2179-1309 • Ano 35•ñ } 110 \bullet \text { Jan./Abr. } 2020\end{array}$} \\
\hline
\end{tabular}


3 - CLAREZA E COMPREENSÃO

4 - PRECISÃO DA INFORMAÇÃO 5 - LAYOUT
Informação concisa

Terminologias apropriadas

1

Integração dos conceitos reflete o entendimento

1 do conceito

Informação correta

1

Mapa em uma página

1

Fácil leitura e interpretação

Hierarquias claras

Demonstra habilidades criativas

TOTAL

Fonte: BHUSNURMATH et al. (2017)

\section{RESULTADOS}

\section{Caracterização dos Estudantes}

Os questionários foram disponibilizados no formulário do Google Drive e foram obtidas as respostas de 64 estudantes. A amostra do estudo foi constituída por $89 \%$ de sujeitos do sexo feminino e $11 \%$ do sexo masculino.

No início do semestre os estudantes foram inquiridos sobre posse e uso de recursos tecnológicos e conhecimentos sobre os mapas conceituais. Foi constatado que todos têm pelo menos um dispositivo móvel e conectam-se diariamente à Internet. A grande maioria (93\%) não tem blog, mas todos têm Facebook ou Linkedin. Ao estudar 54\% escolhem o texto em relação aos outros formatos, como áudio ou vídeo. Preferem ainda ler o texto impresso e sublinhar (95\%) em vez de usar o computador ou um dispositivo móvel. A maioria (73\%) opta por estudar só em detrimento de estudar em grupo. Ao longo da sua formação no ensino básico e secundário, $28 \%$ já fizeram mapas conceituais e $9 \%$ mapas mentais, tendo $64 \%$ respondido que não tiveram contato com nenhuma das técnicas.

\section{Análise dos Mapas Conceituais}

A fim de se avaliar a aprendizagem alcançada, os mapas conceituais elaborados pelos estudantes foram analisados conforme os critérios descritos. A média geral obtida por meio da análise dos mapas conceituais foi de 13,6 pontos ou $68 \%$ (Tabela 2).

Tabela 2 - Resultados da análise dos mapas conceituais de acordo com os critérios utilizados

\begin{tabular}{lcc}
\hline CRITÉRIOS & $\begin{array}{c}\text { MÉDIA OBTIDA/ } \\
\text { TOTAL }\end{array}$ & $\begin{array}{c}\text { PERCENTAGEM } \\
\text { OBTIDA/TOTAL }\end{array}$ \\
\hline 1- ORGANIZAÇÃO & $3,6 / 4,0$ & $18 / 20$ \\
2- INTERCONECTIVIDADE & $3,0 / 7,0$ & $15 / 35$ \\
3- CLAREZA E COMPREENSÃO & $3,0 / 4,0$ & $15 / 20$ \\
4- PRECISÃO DA INFORMAÇÃO & $1,0 / 1,0$ & $5 / 5$ \\
5- LAYOUT & $3,0 / 4,0$ & $15 / 20$ \\
\hline TOTAL & $13,6 / 20,0$ & $68 / 100$ \\
\hline
\end{tabular}

Fonte: Elaborada pelas autoras. 
A análise dos mapas conceituais apontou que no aspecto da Organização (1) 70\% dos mapas conceituais elaborados pelos estudantes apresentaram estrutura em rede e conceitos adequadamente organizados, indicando elevado desempenho cognitivo, entretanto $30 \%$ dos mapas apresentaram número insuficiente dos conceitos, ocasionando mapas incompletos e com deficiências no conteúdo. Neste critério, a média obtida foi de 3,6 pontos. No aspecto da Interconectividade (2), 40\% dos mapas apresentaram as ligações entre conceitos em diferentes segmentos ou domínios do mapa conceitual. No aspecto da Clareza e Compreensão (3), 75\% dos mapas apresentaram conceitos relevantes, com terminologias apropriadas e corretas. Quanto à Precisão da Informação (4), todos os mapas apresentaram informações corretas. No que concerne ao Layout (5), todos os mapas apresentaram-se em uma página e a sua maioria com hierarquia clara. Neste critério a média obtida foi de 3 pontos.

\section{As Percepções dos Estudantes Sobre os Mapas Conceituais}

Ao fim das atividades os estudantes responderam a um questionário sobre suas percepções em relação à abordagem aplicada (Tabela 3).

Tabela 3 - Resultado das percepções dos estudantes sobre os mapas conceituais

\begin{tabular}{|c|c|c|c|c|}
\hline Item & $\begin{array}{l}\text { Discordância } \\
\text { (\%) }\end{array}$ & $\begin{array}{c}\text { Neutro } \\
\text { (\%) }\end{array}$ & $\begin{array}{c}\text { Concordância } \\
\text { (\%) }\end{array}$ & Média \\
\hline $\begin{array}{l}\text { Nas aulas, as atividades com mapas conceituais } \\
\text { em grupo melhoraram a minha aprendizagem. }\end{array}$ & 6 & 22 & 72 & 3,71 \\
\hline $\begin{array}{l}\text { Fazer mapas conceituais é uma boa } \\
\text { oportunidade para aprender com os meus } \\
\text { colegas. }\end{array}$ & 5 & 17 & 78 & 3,85 \\
\hline $\begin{array}{l}\text { Ao fazer os mapas conceituais, eu organizei as } \\
\text { minhas ideias a partir das leituras e discussões } \\
\text { com os meus colegas. }\end{array}$ & 2 & 5 & 93 & 4,08 \\
\hline $\begin{array}{l}\text { Os mapas conceituais ajudaram-me a lembrar } \\
\text { os conceitos estudados. }\end{array}$ & 3 & 11 & 83 & 4,03 \\
\hline $\begin{array}{l}\text { Foi fácil entender o que são os mapas } \\
\text { conceituais. }\end{array}$ & 3 & 23 & 74 & 3,87 \\
\hline $\begin{array}{l}\text { Foi fácil criar os mapas conceituais no Cmap } \\
\text { Tools ou Cmap Cloud }\end{array}$ & 19 & 33 & 45 & 3,32 \\
\hline $\begin{array}{l}\text { Os mapas conceituais são uma forma } \\
\text { interessante para representar o que sei. }\end{array}$ & 0 & 9 & 91 & 4,07 \\
\hline $\begin{array}{l}\text { Auxilia-me a tornar mais claro o conhecimento } \\
\text { sobre o tema estudado. }\end{array}$ & 2 & 16 & 82 & 3,94 \\
\hline
\end{tabular}

Fonte: Elaborada pelas autoras.

Para a maioria dos estudantes foi fácil compreender o que são mapas conceituais (74\%), mas somente $45 \%$ consideraram ter sido fácil criar mapas conceituais no Cmap Tools ou Cmap Cloud. Os mapas conceituais ajudaram a lembrar os conceitos estudados (86\%) e a organizar as ideias com os colegas em grupo (94\%). Criar um mapa conceitual em grupo melhorou a aprendizagem dos estudantes (72\%) e é uma boa oportunida- 
de para aprender com os colegas (78\%). Reconheceram que o mapa conceitual é uma forma interessante para representar o que sabem (91\%) e ajuda a tornar mais claro o conhecimento sobre o tema estudado (82\%).

\section{Aspectos Positivos e Negativos da Abordagem na Perspectiva dos Estudantes}

De acordo com as questões de resposta aberta e após a análise de conteúdo, os estudantes reportaram os aspectos positivos e negativos da abordagem (Tabela 4).

Tabela 4 - Aspectos positivos e negativos da abordagem de acordo com os estudantes

\begin{tabular}{clcc}
\hline \multicolumn{1}{c}{ Item } & Frequência $(\boldsymbol{n}=\mathbf{6 4})$ & \% \\
\hline Aspectos positivos & Uso do software em sala de aula & 40 & 63 \\
& Torna a aprendizagem mais fácil & 24 & 38 \\
& Aulas mais dinâmicas & 22 & 35 \\
& Participação dos estudantes & 9 & 14 \\
Aspectos negativos & Dificuldades com o software & 3 & 4 \\
\hline
\end{tabular}

Fonte: Elaborada pelas autoras.

Sobre a sua experiência com os mapas conceituais, os estudantes apontaram os seguintes aspectos que mais gostaram:

a) o uso das ferramentas tecnológicas (63\%): O uso da tecnologia agradara-me bastante; o facto de podermos utilizar tecnologias.

b) por ser uma metodologia que facilita a aprendizagem (38\%): A experiência é enriquecedora e faz com que sejamos mais autônomos, percebendo melhor a matéria; devido ao nivel de conhecimento que se obtém.

c) por tornarem as aulas mais dinâmicas (35\%): Da dinâmica que as aulas tomaram; Não encher as aulas com muita teoria.

d) ter proporcionado uma maior participação dos estudantes (14\%). O que mais me agradou foi o fato de ter dado uma grande importância à participação do aluno.

\section{Aspectos Negativos da Abordagem na Perspectiva dos Estudantes}

Sobre os aspectos que não gostaram, os estudantes relataram a dificuldade de se trabalhar com as tecnologias (4\%): por vezes é complicado trabalhar com recursos que desconhecemos.

\section{DISCUSSÃO}

Neste estudo, a grande maioria dos estudantes considerou que os mapas conceituais ajudaram a melhorar a sua aprendizagem, tal fato sendo demonstrado na qualidade dos mapas elaborados. Os mapas conceituais representaram um importante potencial aos estudantes para representar o seu conhecimento e para avaliar a sua aprendizagem (ALMEIDA; MOREIRA, 2008). 
Ao construírem os mapas conceituais a partir da análise de um artigo científico, os estudantes puderam usufruir da vantagem proporcionada pelo método como facilitador da aprendizagem, e também como uma ferramenta útil para sistematizar as informações presentes no texto acadêmico.

Os alunos indicaram ainda que as atividades com os mapas os auxiliaram na organização dos conceitos e que tornaram mais fácil a compreensão do conteúdo abordado no artigo científico, revelando-se, portanto, uma ferramenta que facilita a interpretação do conteúdo viabilizando o processo de aprendizagem.

Entre os critérios avaliados nos mapas conceituais elaborados pelos estudantes, a interconectividade apresentou um índice mais baixo, indicando as dificuldades encontradas para estabelecer as ligações entre os conceitos. Em estudos similares, problemas na categorização de conceitos e seleção adequada das suas ligações foram também algumas dificuldades observadas pelos estudantes durante a produção dos mapas conceituais (FARIAS; FARIAS, 2016; GONZÁLEZ et al., 2008).

Apesar de os estudantes reportarem no questionário de caracterização que preferiam estudar sozinhos, após a experiência com mapas conceituais reconheceram que as atividades em grupo facilitaram a organização do conhecimento e a aprendizagem. Afirmaram também que se tornaram mais participativos e que a estratégia incentivou a sua autonomia na construção do conhecimento. Dessa forma, os mapas conceituais elaborados em grupo permitiram propiciar um espaço para a discussão e partilha de ideias. Tal fato é também indicado em outros estudos nos quais os estudantes criaram os mapas conceiuais de forma colaborativa e obtiveram melhores resultados na aprendizagem (ADDAE; WILSON; CARRINGTON 2012; COGO et al., 2009; KINCHIN; HAY, 2005). Além disso, o professor esteve sempre presente nas atividades para o esclarecimento de dúvidas. Notamos neste estudo que a presença do professor durante as atividades com mapas conceituais foi de fundamental importância, pois Ihe possibilitou acompanhar e auxiliar os estudantes no processo de construção dos mapas conceituais. O professor pôde verificar as dificuldades e solucionar dúvidas e equívocos apresentados pelos estudantes durante as atividades. Tal fato é verificado por um índice muito baixo de estudantes que reportaram dificuldades com a ferramenta (4\%).

Um outro fator que causou um impacto positivo na atividade foi permitir o uso de tecnologias para a construção dos mapas conceituais. Neste estudo, os estudantes (63\%) mostraram-se favoráveis ao uso das tecnologias nas atividades. De acordo com suas respostas, um dos aspectos positivos da abordagem foi o de "poder usar as tecnologias. Nesse sentido, os mapas conceituais associados à tecnologia promovem maior participação e envolvimento dos estudantes. Estudos semelhantes também destacaram a preferência dos estudantes em criar os mapas conceituais com o apoio digital por tornar as atividades mais motivadoras e despertar a criatividade (ADDAE; WILSON; CARRINGTON, 2012; ALMEIDA; MOREIRA, 2008; ERDEM; YILMAZ; OSKAY, 2009; LEAUBY; SZABAT; MAAS, 2010). 


\section{CONSIDERAÇÕES FINAIS}

Mapas conceituais são ferramentas utilizadas muitas vezes para representar os conteúdos aprendidos em aula. Neste estudo os alunos utilizaram os mapas conceituais com o apoio do software para a análise de um artigo científico.

As leituras de artigos científicos são práticas constantes no contexto acadêmico e que devem ser inseridas no cotidiano dos estudantes desde os primeiros anos da vida universitária. A adoção de ferramentas que facilitem a aprendizagem é essencial para incentivar o hábito da leitura de artigos científicos, além de favorecer a sua compreensão.

Os mapas conceituais baseados no artigo científico foram criados no Cmaps Tool e demonstraram ser uma eficiente ferramenta para a promoção da aprendizagem. Ao resultarem em uma representação gráfica, os mapas conceituais facilitam a visualização do conhecimento adquirido, tornando evidente o nível de compreensão dos estudantes sobre o conteúdo presente no artigo científico. Além disso, os mapas conceituais foram bem aceitos pelos estudantes durante as atividades acadêmicas e possibilitaram ao professor o acompanhamento e a avaliação da aprendizagem, bem como o auxiliaram na detecção de dificuldades nos conteúdos. Além disso, são ferramentas que ao serem trabalhadas em grupo incentivam uma maior participação dos estudantes nas atividades. Segundo os estudantes, as tecnologias tornaram as atividades mais interativas, dinâmicas e incentivaram o desenvolvimento da sua autonomia no processo de aprendizagem. Sobre as dificuldades encontradas por alguns estudantes foi apontada a falta de habilidade na utilização do software. Tal dificuldade foi minimizada pela presença do professor para o acompanhamento dos estudantes durante as atividades, que permitiu auxiliá-los na elucidação de dúvidas de interpretação do texto científico e no uso do software. Diante das várias formas de se alcançar a aprendizagem que essa ferramenta viabiliza, além de permitir o desenvolvimento de habilidades como o pensamento crítico, o trabalho colaborativo e a comunicação, torna-se importante trazer à sala de aula a oportunidade de levar o estudante universitário a um crescimento mais efetivo, que favoreça seu progresso profissional.

\section{AGRADECIMENTOS}

Aos estudantes participantes deste estudo, ao LabTE e CEIS20 da Universidade de Coimbra pelo fomento à pesquisa.

\section{REFERÊNCIAS}

ADDAE, Jonas Innies; WILSON, Jacqueline I.; CARRINGTON, Christine. Students' perception of a modified form of PBL using concept mapping. Medical Teacher, [s.I.], v. 34, n. 11, p. 756-762, nov. 2012.

ALMEIDA, Voltaire; MOREIRA, Marco. Antônio. Mapas conceituais no auxílio à aprendizagem significativa de conceitos da óptica física. Revista Brasileira de Ensino de Física, São Paulo, v. 30, n. 4, p. 1-7, 2008.

AMADO, João. Manual de investigação qualitativa em educação. Coimbra: Imprensa da Universidade de Coimbra, 2014. 427p.

ARAÚJO, Mauro. Sérgio Teixeira; FORMENTON, Ricardo. Utilização de mapa conceitual como ferramenta de análise de trabalhos científicos. Holos, [s.I.], v. 1, p. 171-181, 2015. DOI: 10.15628/holos.2015.2130

ASAN, Askin. Concept mapping in science class: a case study of fifth grade students. Journal of Educational Technology \& Society, [s.I.], v. 10, n. 1, p. 186-195, 2007. 
BES PIÁ, Amparo; BLASCO-TAMARITI, Encarna; MUÑOZ-PORTERO, María José. Different applications of concept maps in higher education. Journal of Industrial Engineering and Management, [s.I.], v. 4, p. 81102, 2011.

BITTENCOURT, Greicy Kelly Gouveia Dias et al. Concept maps of the graduate programme in nursing: experience report. Revista Gaúcha de Enfermagem. Porto Alegre, v. 34, n. 2, p. 172-176, 2013.

BHUSNURMATH, Shivayogi et al. Concept map as an adjunct tool to teach pathology. Indian Journal of Pathology and Microbiology, [s.I.], v. 60, n. 2, p. 226-231, 2017.

CAÑAS, Alberto et al. CmapTools: A Knowledge Modeling and Sharing Environment. In: INTERNATIONAL CONFERENCE ON CONCEPT MAPPING, 1., 2004, Pamplona. Proceedings. Pamplona, Spain: Universidad Pública de Navarra, 2004. p. 125-133.

CAÑAS, Alberto; NOVAK, Joseph. Concept mapping using cmap tools to enhance meaningful learning. In: OKADA, Alexandra; BUCKINGHAM SHUM, Simon; SHERBORNE, Tony (coord.). Knowledge cartography: Software tools and mapping techniques. London, UK: Springer-Verlag, 2008. p. 25-46.

CARABETTA JÚNIOR, Valter. A utilização de mapas conceituais como recurso didático para a construção e inter-relação de conceitos. Revista Brasileira de Educação Médica, Rio de Janeiro, v. 37, n. 3, p. 441-447, 2013.

CARR-LOPEZ, Sian et al. The utility of concept maps to facilitate higher-level learning in a large classroom setting. American Journal of Pharmaceutical Education, [s.I.], v. 78, n. 9, p. 1-7, nov. 2014.

CARVALHO, Ana Amélia; PESSOA, Teresa. Políticas educativas TIC en Portugal. Campus Virtuales, [s.I.], v. 1, p. 93-104, 2012.

CHAN, Zenobia. A qualitative study on using concept maps in problem-based learning. Nurse Education in Practice, [s.l.], v. 24, p. 70-76, May 2017.

CHEN, Shiah-Lian et al. Effects of Concept Map Teaching on Students' Critical Thinking and Approach to Learning and Studying. Journal of Nursing Education, [s.I.], v. 50, n. 8, p. 466-469, 2011.

COGO, Ana Luísa Petersen et al. Avaliação de mapas conceituais elaborados por estudantes de enfermagem com o apoio de software. Texto \& Contexto Enfermagem, Florianópolis, v. 18, n. 3, p. 482-488, 2009. COUTINHO, Clara Pereira. Metodologia de investigação em ciências sociais e humanas: teoria e prática. 2. ed. Coimbra: Almedina, 2013. 412p.

DUARTE, Enios Carlos; LOUREIRO, Ana Claudia; ZUKOWSKY-TAVARES, Cristina. Challenges and weaknesses in the use of concept maps as a learning strategy in undergraduate health programs. Knowledge Management \& E Learning, [s.I.], v. 9, n. 3, p. 380-391, 2017.

ERDEM, Emine; YILMAZ, Ayhan; OSKAY, Özge Özyalçın. The effect of concept mapping on meaningful learning of Atom and bonding. Procedia - Social and Behavioral Sciences, [s.I.], v. 1, n. 1, p. 1586-1590, 2009.

ERDOGAN, Yavuz. Paper-based and computer-based concept mappings: The effects on computer achievement, computer anxiety and computer attitude. British Journal of Educational Technology, [s.l.], v. 40, n. 5, p. 821-836, 2009.

FARIAS, Maria Giovanna Guedes; FARIAS, Gabriela Belmont. Aplicação de mapas conceituais como ferramentas didático-pedagógicas na área de recursos e serviços de informação. Biblios: Journal of Librarianship and Information Science, [s.I.], n. 63, p. 13-27, 2016.

GARCíA, Fermín Maria González et al. Aplicación de mapas conceptuales en ingeniería en computación para evaluar aprendizaje significativo. Revista de Docencia Universitaria, [s.I.], v. 10, p. 459-475, 2012.

GERDEMAN, Jaime L.; LUX, Kathleen; JACKO, Jean. Using concept mapping to build clinical judgment skills. Nurse Education in Practice, [s.l.], v. 13, n. 1, p. 11-17, jan. 2013.

GONZÁLEZ, Hilda Leonor et al. Mediated learning experience and concept maps: a pedagogical tool for achieving meaningful learning in medical physiology students. AJP: Advances in Physiology Education, [s.l.], v. 32, n. 4, p. 312-316, 2008.

GREENBERG, Rochelle Kaplan; WILNER, Neil. Using concept maps to provide an integrative framework for teaching the cost or managerial accounting course. Journal of Accounting Education, [s.l.], v. 33, n. 1, p. 16-35, 2015.

HAY, David; KINCHIN, Ian; LYGO-BAKER, Simon. Making learning visible: the role of concept mapping in higher education. Studies in Higher Education, [s.I.], v. 33, n. 3, p. 295-311, 2008.

HO, Veronica; KUMAR, Rakesh; VELAN, Gary. Online testable concept maps: benefits for learning about the pathogenesis of disease. Medical Education, [s.I.], v. 48, n. 7, p. 687-697, 2014.

KASSAB, Salah et al. Generalisability theory analyses of concept mapping assessment scores in a problem-based medical curriculum. Medical Education, [s.I.], v. 50, p. 730-737, 2016. 
KATILIUTE, Egle; DAUNORIENE், Asta. Toward meaningful learning in economics and management studies using concept maps in a quality management course. Economics \& Management, [s.l.], v. 16, p. 758-765, 2011.

KINCHIN, Ian; HAY, David; ADAMS, Alan. How a qualitative approach to concept map analysis can be used to aid learning by illustrating patterns of conceptual development. Educational Research [s.l.], v. 42, p. 43-57, 2000.

KINCHIN, Ian; HAY, David. Using concept maps to optimize the composition of collaborative student groups: a pilot study. Journal of Advanced Nursing, [s.I.], v. 51, n. 2, p. 182-187, 2005.

LEAUBY, Bruce; SZABAT, Kathryn; MAAS, Jayne. Concept Mapping-an empirical study in introductory financial accounting. Accounting Education, [s.I.], v. 19, n. 3, p. 279-300, 2010.

MARÉE, Ton; VAN BRUGGEN, Jan; JOCHEMS, Wim. Effective self-regulated science learning through multimedia-enriched skeleton concept maps. Research in Science \& Technological Education, [s.l.], v. 31, n. 1, p. 16-30, 2013.

MIRANDA, Luísa; MORAIS, Carlos. Mapas conceptuais como estratégia de ensino e aprendizagem. In: CONGRESSO INTERNACIONAL GALEGO-PORTUGUÊS DE PSICOPEDAGOGIA, 10., 2009, Braga. Actas [...]. Braga: CIGPP, 2009. p. 3.101-3.110.

MOK, Cecilia; WHITEHILL, Tara; DODD, Barbara. Concept map analysis in the assessment of speech-language pathology students' learning in a problem-based learning curriculum: A longitudinal study. Clinical Linguistics \& Phonetics, [s.I.], v. 28, p. 64-82, 2014.

MOTTA, Marcelo Souza. Formação inicial do professor de matemática no contexto das tecnologias digitais. Revista Contexto e Educação, ljuí, v. 32, n. 102, p. 170-204, 2017.

NESBIT, John; ADESOPE, Olusola. Learning with concept and knowledge maps: A meta-analysis. Review of Educational Research, [s.I.], v. 76, n. 3, p. 413-448, 2006.

NOVAK, Joseph. Learning, creating, and using knowledge: concept maps as facilitative tools in schools and corporations. Journal of e-Learning and Knowledge Society, [s.I.], v. 6, p. 21-30, 2010.

NOVAK, Joseph. The promise of new ideas and new technology for improving teaching and learning. Cell Biology Education, [s.I.], v. 2, p. 122-132, 2003.

NOVAK, Joseph; CAÑAS, Alberto. The origins of the concept mapping tool and the continuing evolution of the tool. Information Visualization Journal, [s.I.], v. 5, n. 3, p. 175-184, 2006.

NOVAK, Joseph; GOWIN, Bob. Learning How to Learn. New York: Cambridge University, 1984.

OLIVEIRA, Eloiza Silva Gomes; SILVA, Marcos Antônio. Mapas conceituais e aprendizagem no ciberespaço: uma reflexão sobre didática e partituras musicais. Educação Temática Digital, Campinas, v. 8, n. 1, p. 7289, 2006.

RAHNAMA, Farideh; MARDANI-HAMOOLEH, Marjan. Iranian nursing students' perceptions regarding use of concept mapping: a content analysis. Research \& Development in Medical Education, [s.l.], v. 6, n. 1, p. 45-50, 2017.

RITCHHART, Ron; TURNER, Terri; HADAR, Linor. Uncovering students' thinking about thinking using concept maps. Metacognition and Learning, [s.I.], v. 4, n. 2, p. 145-159, 2009.

RUIZ-MORENO, Lidia et al. Mapa conceitual: ensaiando critérios de análise. Ciência \& Educação, Bauru, v. 13, n. 3, p. 453-463, 2007.

SANTOS, Gelson Ribeiro; QUEIROZ, Salete Linhares. Leitura e interpretação de artigos científicos por alunos de graduação em química. Ciência \& Educação, Bauru, v. 13, n. 2, p. 193-209, 2007.

SENITA, Julie. The use of concept maps to evaluate critical thinking in the clinical setting. Teaching and Learning in Nursing, [s.I.], v. 3, n. 1, p. 6-10, 2008.

SILVA, Janice Henriques et al. O ensino-aprendizagem da anatomia humana: avaliação do desempenho dos alunos após a utilização de mapas conceituais como uma estratégia pedagógica. Ciências \& Educação, Bauru, v. 24, n. 1, p. 95-110, 2018.

SONNLEITNER, Philip et al. The Genetics Lab: acceptance and psychometric characteristics of a computer-based microworld assessing complex problem solving. Psychological Test and Assessment Modeling, [s.l.], v. 54, n. 1, p. 54-72, 2012.

SOUZA, Nadia Aparecida; BORUCHOVITCH, Evely. Mapas conceituais e avaliação formativa: tecendo aproximações. Educação e Pesquisa, São Paulo, v. 36, n. 3, p. 795-810, 2010.

TSAI, Chin Chung; LIN, Suny; YUAN, Shi-Min. Students' use of web-based concept map testing and strategies for learning. Journal of Computer Assisted Learning, [s.I.], v. 17, n. 1, p. 72-84, 2008. 


\section{\& Contexto}

Mapa Conceitual Como Ferramenta de Aprendizagem no Ensino Superior

VACEK, Jenny. Using a conceptual approach with concept mapping to promote critical thinking. Journal of Nursing Education, [s.I.], v. 48, n. 1, p. 45-48, 2009.

VERONESE, Carolina et al. A randomized pilot study of the use of concept maps to enhance problem-based learning among first-year medical students. Medical Teacher, [s.I.], v. 35, n. 9, p. 1.478-1.484, 2013.

WANG, Minhong et al. The use of web-based collaborative concept mapping to support group learning and interaction in an online environment. The Internet and Higher Education, [s.I.], v. 34, p. 28-40, 2017.

WILGIS, Michele; MCCONNELL, Joy. Concept Mapping: An educational strategy to improve graduate nurses' critical thinking skills during a hospital orientation program. The Journal of Continuing Education in Nursing, [s.l.], v. 39, n. 3, p. 119-126, 2008.

WU, Sheng-Vi; CHEN, Sherry Y.; HOU, Huei-Tse. Exploring the interactive patterns of concept map-based online discussion: a sequential analysis of users' operations, cognitive processing, and knowledge construction. Interactive Learning Environments, [s.I.], v. 24, n. 8, p. 1.778-1.794, 2016.

YEN, Jung-Chuan; LEE, Chun-Yi; CHEN, I-Jung. The effects of image-based concept mapping on the learning outcomes and cognitive processes of mobile learners. British Journal of Educational Technology, [s.l.], v. 43, n. 2, p. 307-320, 2011. 